\title{
Case report: tumor-treating fields prolongs IDH-mutant anaplastic astrocytoma progression-free survival and pathological evolution to glioblastoma
}

\author{
Yi $\operatorname{Lin}^{1} \wedge$, Baoshi Chen²^ \\ ${ }^{1}$ Department of Neuro-Oncology, Cancer Center, Beijing Tiantan Hospital, Capital Medical University, Beijing, China; ${ }^{2}$ Department of \\ Neurosurgery, Beijing Tiantan Hospital, Capital Medical University, Beijing, China \\ Correspondence to: Baoshi Chen. Department of Neurosurgery, Beijing Tiantan Hospital, Capital Medical University, 119 South $4^{\text {th }}$ Ring West Road, \\ Fengtai District, Beijing 100070, China. Email: 13601212538@163.com.
}

\begin{abstract}
Gliomas are one of the most common tumors of the central nervous system, which cause significant morbidity and mortality. Glioblastoma (GBM) is an aggressive and debilitating disease, which is the most common primary brain tumor in adults. Despite optimal surgical resection followed by radiotherapy and adjuvant chemotherapy, prognosis of patients with GBM remain poor. Tumor-treating fields (TTFields), as a novel treatment approach, is being explored through increased clinical application. Herein, we report the case of a 46-year-old man who was diagnosed as anaplastic astrocytoma [World Health Organization (WHO) III grade], IDH1 R132 mutation, IDH2 R172 mutation, and a methylated MGMT promoter, without 1p36 and $19 \mathrm{q} 13$ heterozygosity loss. About 2.5 months after surgery, the patient presented with seizures, aphasia, and memory loss. Following the first-line treatment, TTFields was shown to be a valuable treatment option to control clinical symptom burden. The use of TTFields was shown to prolong progression-free survival and delay the pathological upgradation to glioblastoma. Notably, combined TTFields and chemotherapy might be beneficial in terms of risk reduction for pathological upgradation. To the our current knowledge, this is the first case report that attempts to offer possible strategies based on TTFields toward delaying pathological upgradation from anaplastic astrocytoma (WHO III grade) to glioblastoma.
\end{abstract}

Keywords: Isocitrate dehydrogenase; glioblastoma; anaplastic astrocytoma; tumor-treating fields (TTFields); case report

Submitted Aug 10, 2021. Accepted for publication Dec 14, 2021.

doi: $10.21037 /$ atm-21-4760

View this article at: https://dx.doi.org/10.21037/atm-21-4760

\section{Introduction}

Gliomas are one of the most common tumors of the central nervous system, which cause significant morbidity and mortality (1). Mutations in the isocitrate dehydrogenase (IDH) 1 or 2 genes are frequently detected in lower [World Health Organization (WHO) II/III] grade tumors but are typically absent in classical glioblastoma. The IDH gene has also been identified as a metabolic driver enzyme in the development of several tumor types $(1,2)$. Mutations of IDH lead to loss of catalyzing interconversion of isocitrate to $\alpha$-ketoglutarate $(\alpha-K G)$, alter the cellular epigenetic profile, inhibit differentiation, and initiate tumorigenesis (3). Mutation of IDH1 is detected in 70-90\% of low-grade gliomas and upgraded glioblastomas (4). Notably, the diagnostic importance of IDH mutational status was recently recognized in an update of recommended grading

\footnotetext{
^ ORCID: Yi Lin, 0000-0001-7523-2250; Baoshi Chen, 0000-0003-2809-7759.
} 

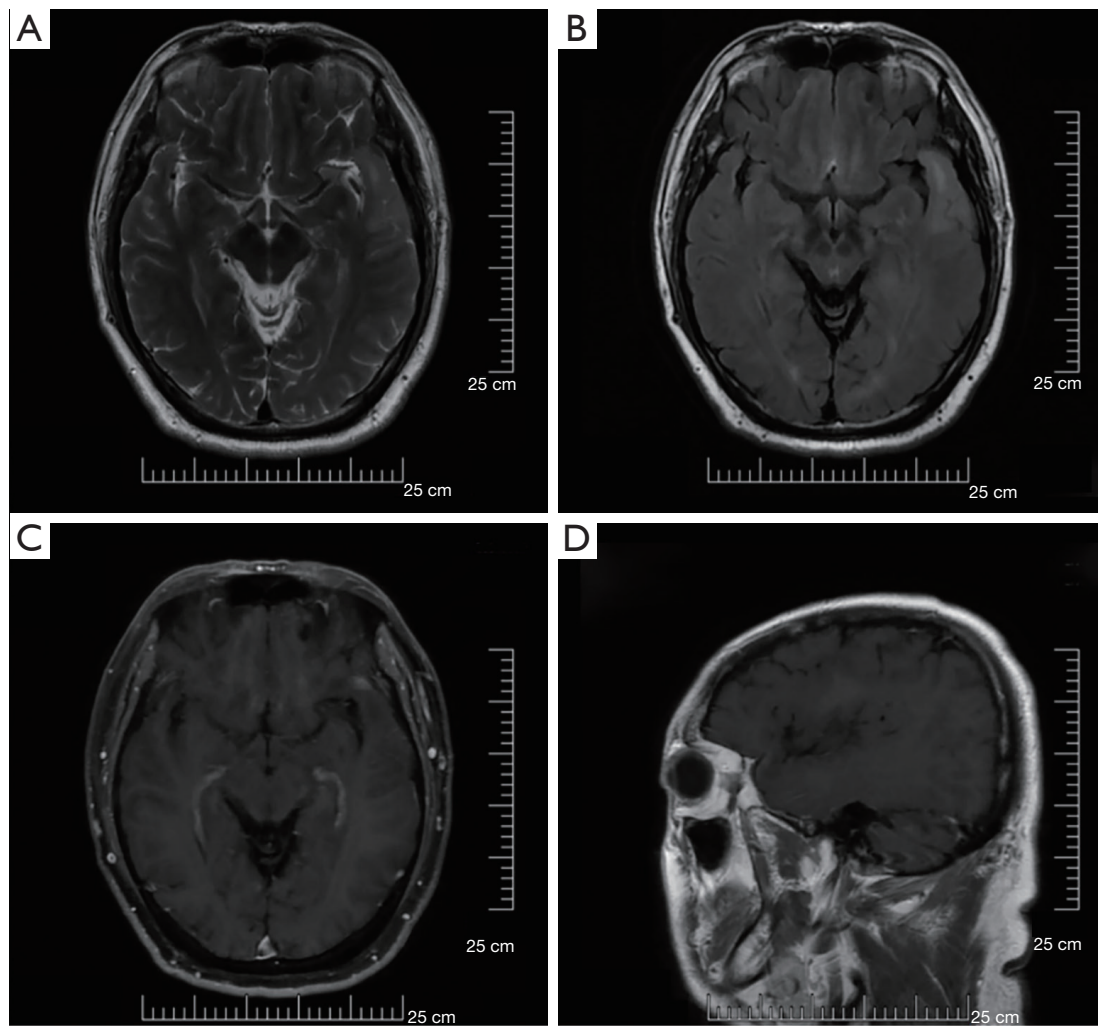

Figure 1 Preoperative MRI showing (A) a slightly higher signal in T2 sequence, (B) high signal in FLAIR sequence, (C,D) enhanced signal. MRI, magnetic resonance imaging; FLAIR, fluid-attenuated inversion recovery.

criteria and terminologies for IDH-mutant astrocytomas $(5,6)$. Astrocytomas with an IDH-mutation that exhibit microvascular proliferation or necrosis or cyclin-dependent kinase inhibitor $(\mathrm{CDKN}) 2 \mathrm{~A} / \mathrm{B}$ homozygous deletion, or any combination of these features, have been formerly considered as "glioblastoma, IDH-mutant, WHO grade IV" (7). Herein, we have reported a case of an IDH-mutant anaplastic astrocytoma upgraded to glioblastoma, in which the use of tumor-treating fields (TTFields) prolonged progression-free survival. We have also discussed in detail what is known to date about the CDKN2A/B homozygous deletion and TTFields, and how TTFields have been synergistically used with other treatments. We present the following article in accordance with the CARE reporting checklist (available at https://dx.doi.org/10.21037/atm-214760).

\section{Case presentation}

A 46-year-old previously healthy man presented with progressive memory loss for 6 months. The patient had no significant prior history or familial history of cancer. $\mathrm{He}$ experienced facial and limb convulsions, and a 10-minute episode of unconsciousness 1 month ago, which he was unable to recall after awaking.

On further inquiry, he denied nausea, vomiting, or headaches, visual or speech disturbances, limb paresthesia, or weakness. His past medical history was a trauma to the left abdomen and right thigh 10 years ago, for which he had undergone surgical treatment. The patient had a history of hypertension for 1 year, and his blood pressure had reached up to $150 / 100 \mathrm{mmHg}$. His blood pressure had been controlled at $130 / 80 \mathrm{mmHg}$ by oral administration of felodipine sustained-release tablets $5 \mathrm{mg}$ once a day. Neurologic examination revealed normal strength, grade 5 power of his extremities, with normal reflexes. The patient's Karnofsky Performance Score (KPS) was 100.

The patient underwent brain magnetic resonance imaging (MRI) with and without contrast, which showed intracranial space-occupying lesions, and enhanced signals in the left frontal lobe, junction area of left frontal lobe, temporal lobe, and parietal lobe (Figure 1). He underwent 

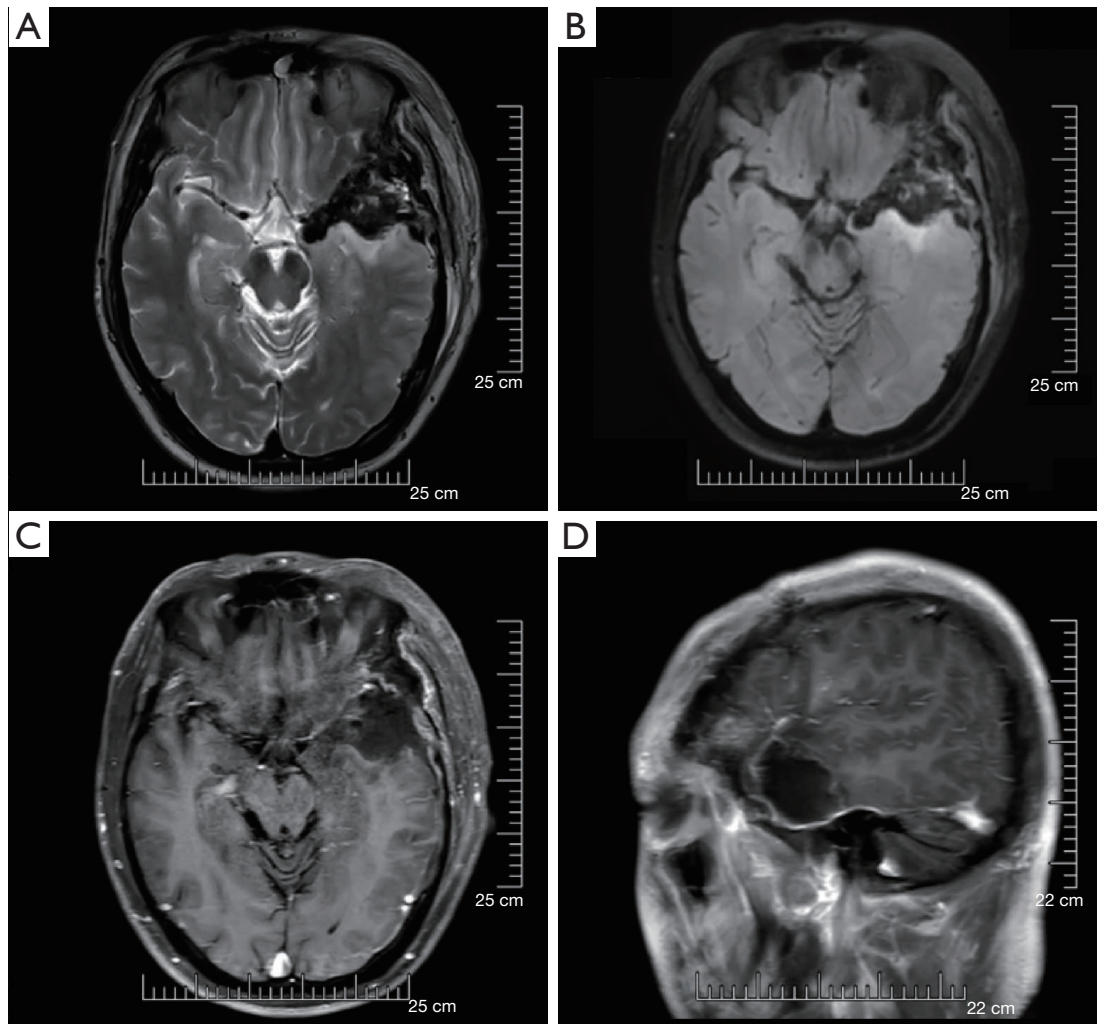

Figure 2 Postoperative MRI showing (A,B) a mixed slightly higher T1T2 signal, (C) a high FLAIR signal, (D) enhanced signal after contrast. MRI, magnetic resonance imaging; FLAIR, fluid-attenuated inversion recovery.

left frontotemporal craniotomy plus artificial dural repair, and postoperative MRI examination showed no residual tumor (Figure 2). The pathology result reported anaplastic astrocytoma (WHO III grade), IDH1 R132 mutation, IDH2 R172 mutation, and a methylated MGMT promoter, without $1 \mathrm{p} 36$ and $19 \mathrm{q} 13$ heterozygosity loss. The patient underwent standard treatment, consisting of radiotherapy of a total dose of $54 \mathrm{~Gy}$ in 30 divided fractions, with concurrent daily temozolomide (TMZ; $75 \mathrm{mg} / \mathrm{m}^{2}$ of body surface area), followed by 3 cycles of adjuvant TMZ $\left(150 \mathrm{mg} / \mathrm{m}^{2}\right)$ for 5 days every 28 days. About 2.5 months after the operation, the patient presented with seizures, aphasia, and memory loss. An MRI examination showed no sign of tumor recurrence (Figure 3). The patient's chemotherapy regimen was changed to cisplatin $\left(80 \mathrm{mg} / \mathrm{m}^{2}\right)$ combined with TMZ $\left(150 \mathrm{mg} / \mathrm{m}^{2}\right)$. His speech function improved after using bevacizumab targeted therapy treatment.

About 4.5 months after the operation, the patient started TTFields (Figure 4). Thus, the patient received chemotherapy and targeted therapy treatment for 3 cycles, the regimen was cisplatin $\left(30 \mathrm{mg} / \mathrm{m}^{2}\right)$, TMZ $\left(150 \mathrm{mg} / \mathrm{m}^{2}\right)$, and bevacizumab $(5 \mathrm{mg} / \mathrm{kg})$. Brain MRI scan showed abnormal signals in the left caudate nucleus and left ventricle, suggesting tumor recurrence (Figure 5). The patient experienced more frequent seizures than before, and his speech was slurred after the seizure. To confirm whether the tumor had recurred, the patient underwent stereotactic biopsy about 10 months after the operation. The pathological results showed glioblastoma. Molecular test results confirmed MGMT promoter methylation, IDH wild type, EGFR amplification, with PTEN and CDKN2A/ B homozygous deletion.

All procedures performed in this study involving human participants were in accordance with the ethical standards of the institutional and/or national research committee(s) and with the Helsinki Declaration (as revised in 2013). Written informed consent was obtained from the patient for publication of this case report and accompanying images. A copy of the written consent is available for review by the editorial office of this journal. 

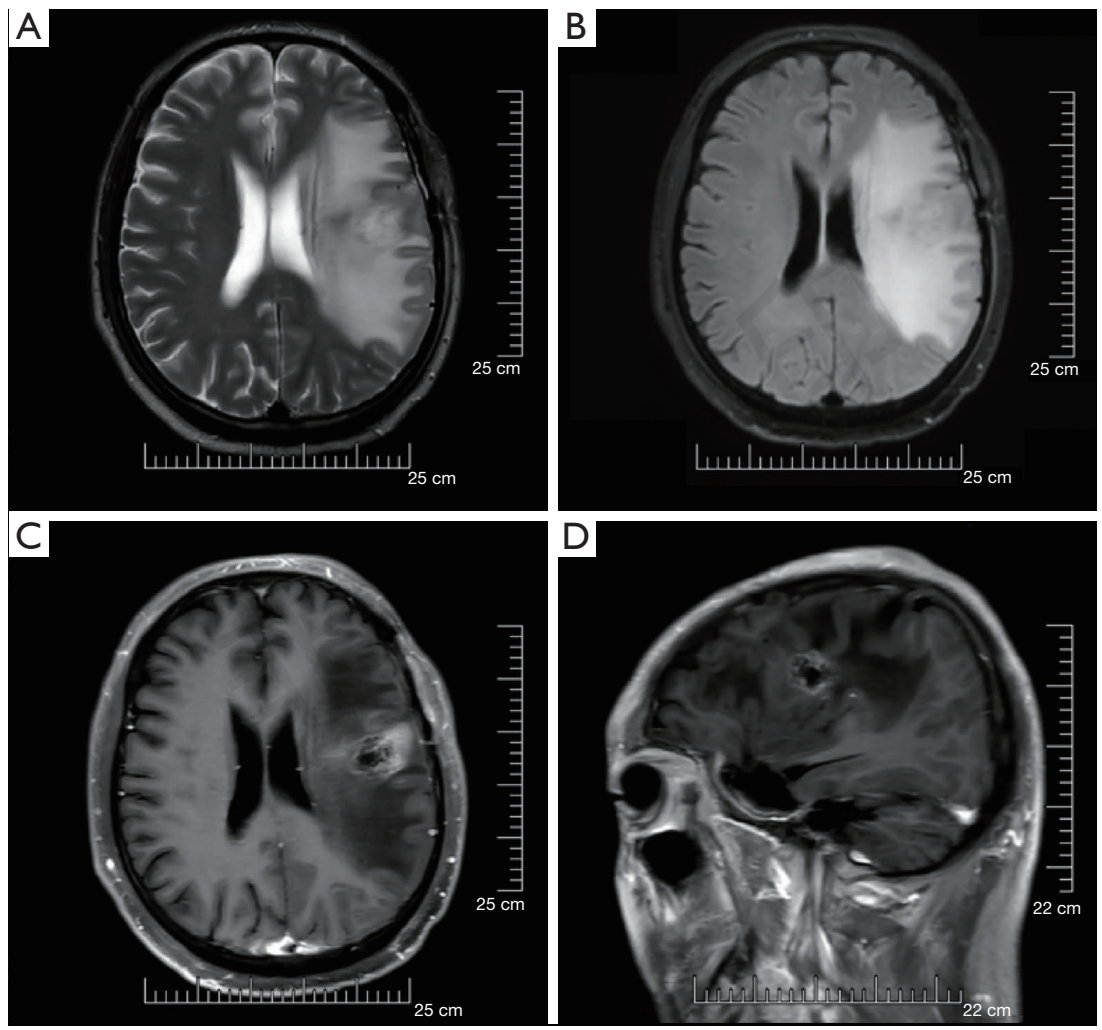

Figure 3 Post-radiation MRI showing (A) a mixed high T2WI signal, (B) a high signal FLAIR, (C,D) a piece of small ring-shaped enhancement. MRI, magnetic resonance imaging; FLAIR, fluid-attenuated inversion recovery.
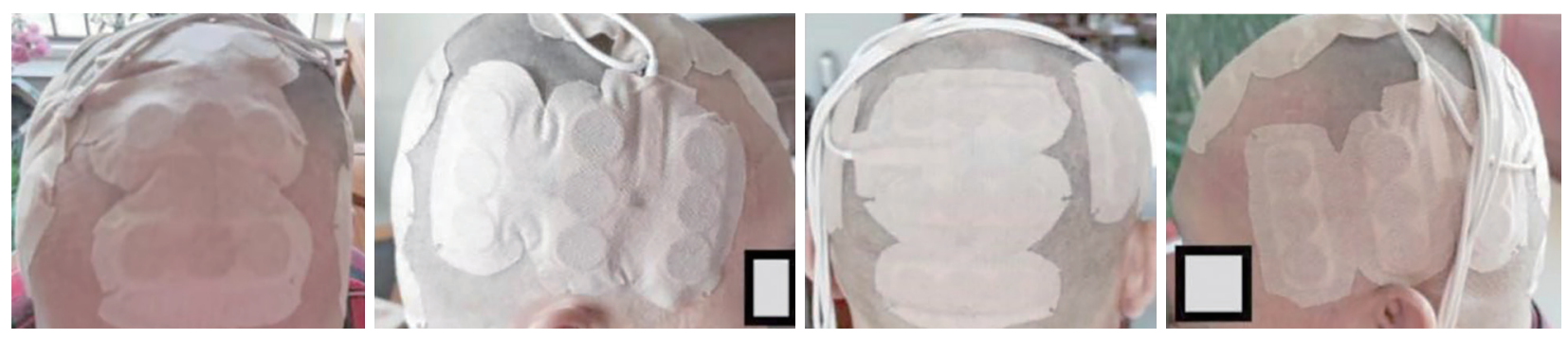

Figure 4 Placement of the tumor-treating fields electrodes.

\section{Discussion}

Gliomas are common intrinsic brain tumors, which are classified into different subtypes by the WHO grades (8). Numerous studies have attempted to identify molecular biomarkers and prognostic indicators in gliomas and stratify these tumors into different grades. Genetic and epigenetic features facilitate a relatively complete grading system and underlying complex biological effect that results in different clinical outcomes. One of the most significant of these driving molecular changes has been the IDH-wild type and
IDH-mutant of adult anaplastic astrocytoma. Assessment of the glioma for IDH mutations was recommended as a required molecular testing for "integrated diagnosis" in the 2016 revised WHO classification of tumors of the central nervous system. In addition to histologic grade, the clinical outcome needs to be described within these IDH categories (9). Current diagnostic, prognostic, and predictive considerations also depend on IDH status (10). With the recent publication of the cIMPACT-NOW update 5, new molecular features are now included in the diagnostic and 

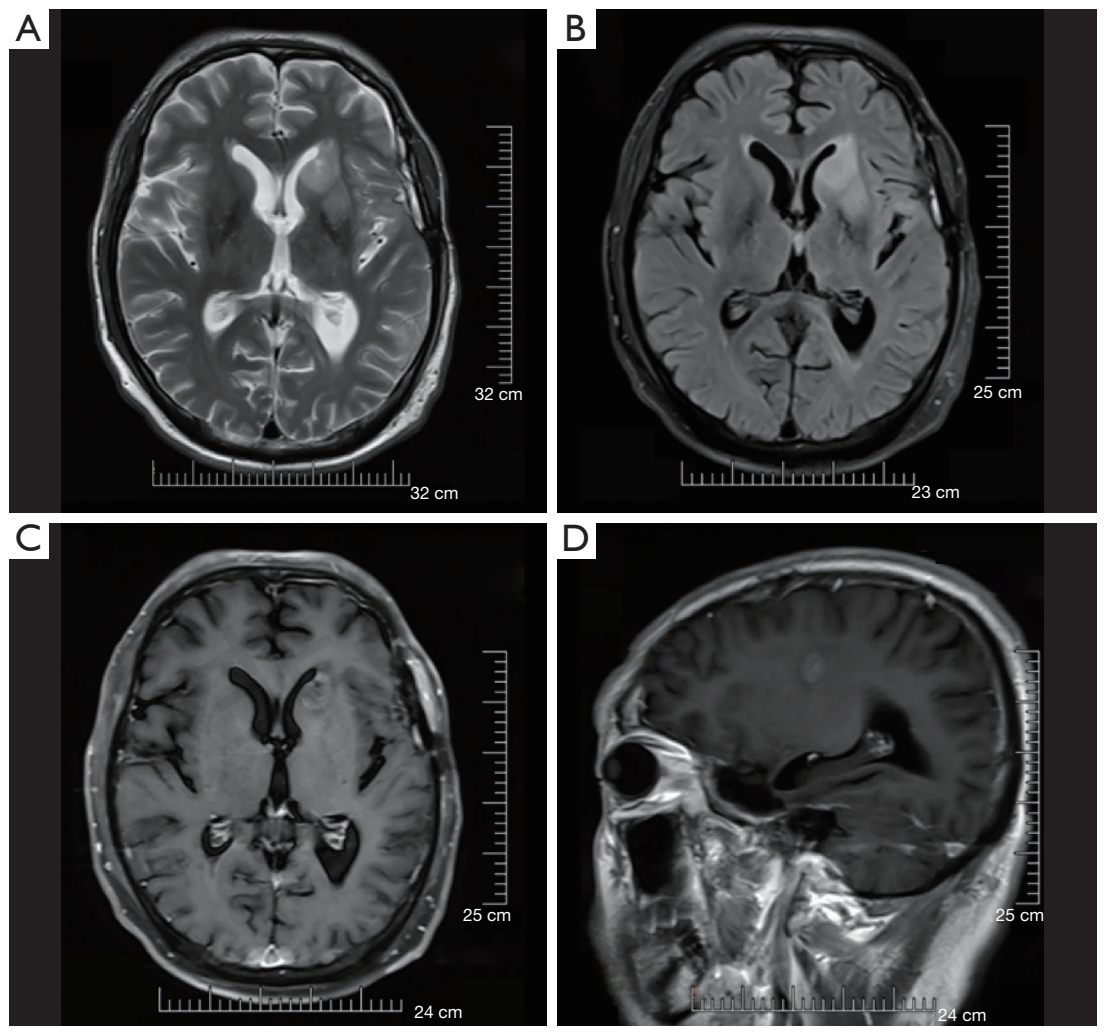

Figure 5 Pre-biopsy MRI showing (A,B) a higher T2 signal, (C) a high signal FLAIR, (D) uneven enhancement and blurred edges. MRI, magnetic resonance imaging; FLAIR, fluid-attenuated inversion recovery.

grading system (7). The update highlighted the homozygous deletion of the cyclin-dependent kinase inhibitor $2 \mathrm{~A} / \mathrm{B}$ $(\mathrm{CDKN} 2 \mathrm{~A} / \mathrm{B})$ gene as a new clinical molecular indicator in IDH-mutant glioma $(11,12)$. Clinical data showed that these tumors behave similarly to glioblastoma (GBM). Therefore, the proper classification of IDH-mutant glioma is increasingly dependent on molecular genetic tests for neuropathological evaluation.

The TTFields have emerged as an additional treatment option by delivering low-intensity (1-3 V/cm), intermediatefrequency $(100-500 \mathrm{kHz})$, alternating electric fields to inhibit tumor cell growth $(13,14)$. A randomized clinical trial analysis of 315 patients with GBM, who had completed standard chemoradiation therapy, showed that adding TTFields to maintenance TMZ chemotherapy significantly prolonged the progression-free survival (PFS) and overall survival (OS) (15). Thus, TTFields was approved by the Food and Drug Administration (FDA) in adjuvant treatment of GBM, based on the positive phase $3 \mathrm{EF}-11$ (recurrent GBM, rGBM) results and EF-14 (newly diagnosed GBM, ndGBM) trials. Notably, TTFields is classified as a category
1 recommendation in the National Comprehensive Cancer Network ${ }^{\circledR}$ guidelines for ndGBM and is recognized as a treatment advancement in clinical cancer care by the American Society of Clinical Oncology (16). China's Glioma Treatment Guidelines [2018] also recommend TTFields for patients with GBM based on level 1 evidence. However, experience of applying TTFields in IDH-mutant anaplastic astrocytoma is very limited (17).

Currently, the standard treatment for anaplastic astrocytoma is based on surgical resection, followed by adjuvant radiotherapy (RT) plus concomitant and maintenance TMZ chemotherapy. Despite the multidisciplinary team (MDT), PFS and OS of IDH-mutant anaplastic astrocytoma were unfavorable because anaplastic astrocytoma almost always upgrades to GBM. A study showed that PFS for GBM was 4.0 months in the TMZ-alone group and 6.7 months in the TTFields-TMZ group (18). Bevacizumab prolongs PFS in ndGBM and rGBM, but was no available evidence supporting IDH-mutant anaplastic astrocytoma with homozygous deletion of the CDKN2A/ B. However, the present case indicated that TTFields in 
combination with maintenance temozolomide treatment can benefit patients with ndGBM who were previously tested as IDH-mutant anaplastic astrocytoma with homozygous deletion of the CDKN2A/B. In addition to the first-line treatment, TTFields prolonged PFS to nearly 10 months and delayed the pathological upgradation to GBM. The patient has expressed a desire to maintain the efficacy of TTFields, and hopes for favorable long-term results. The TTFields is considered a valuable treatment option to control clinical symptom burden. Additionally, there has been reported that the combination of TTFields with proton radiotherapy in treatment of primary GBM, and observed complete radiological response after subtotal resection (STR), chemoradiation, and TTFields. The results also suggested that adjuvant TTFields therapy can structurally affect the residual tumors after subtotal resection (19). The most common TTFields-related adverse event was array-associated skin irritation (13-16). In the present case, no adverse reactions were clinically identified. Given the generally poor outcome of IDH-mutant anaplastic astrocytoma patients with homozygous deletion of the CDKN2A/B, TTFields is increasingly being explored as a novel treatment approach in clinical trials. Overall, ongoing and future trials will evaluate TTFields in other types of glioma and solid tumor brain metastases and explore the potential benefit for patients.

\section{Acknowledgments}

Funding: None.

\section{Footnote}

Reporting Checklist: The authors have completed the CARE reporting checklist. Available at https://dx.doi. org/10.21037/atm-21-4760

Conflicts of Interest: Both authors have completed the ICMJE uniform disclosure form (available at https://dx.doi. org/10.21037/atm-21-4760). The authors have no conflicts of interest to declare.

Ethical Statement: The authors are accountable for all aspects of the work in ensuring that questions related to the accuracy or integrity of any part of the work are appropriately investigated and resolved. All procedures performed in this study involving human participants were in accordance with the ethical standards of the institutional and/or national research committee(s) and with the Helsinki
Declaration (as revised in 2013). Written informed consent was obtained from the patient for publication of this case report and accompanying images. A copy of the written consent is available for review by the editorial office of this journal.

Open Access Statement: This is an Open Access article distributed in accordance with the Creative Commons Attribution-NonCommercial-NoDerivs 4.0 International License (CC BY-NC-ND 4.0), which permits the noncommercial replication and distribution of the article with the strict proviso that no changes or edits are made and the original work is properly cited (including links to both the formal publication through the relevant DOI and the license). See: https://creativecommons.org/licenses/by-nc-nd/4.0/.

\section{References}

1. Tan AC, Ashley DM, López GY, et al. Management of glioblastoma: State of the art and future directions. CA Cancer J Clin 2020;70:299-312.

2. Mirchia K, Richardson TE. Beyond IDH-Mutation: Emerging Molecular Diagnostic and Prognostic Features in Adult Diffuse Gliomas. Cancers (Basel) 2020;12:1817.

3. Korshunov A, Casalini B, Chavez L, et al. Integrated molecular characterization of IDH-mutant glioblastomas. Neuropathol Appl Neurobiol 2019;45:108-18.

4. Viswanath P, Chaumeil MM, Ronen SM. Molecular Imaging of Metabolic Reprograming in Mutant IDH Cells. Front Oncol 2016;6:60.

5. Feraco P, Bacci A, Ferrazza P, et al. Magnetic Resonance Imaging Derived Biomarkers of IDH Mutation Status and Overall Survival in Grade III Astrocytomas. Diagnostics (Basel) 2020;10:247 .

6. Dono A, Wang E, Lopez-Rivera V, et al. Molecular characteristics and clinical features of multifocal glioblastoma. J Neurooncol 2020;148:389-97.

7. Brat DJ, Aldape K, Colman H, et al. cIMPACT-NOW update 5: recommended grading criteria and terminologies for IDH-mutant astrocytomas. Acta Neuropathol 2020;139:603-8.

8. Ostrom QT, Cote DJ, Ascha M, et al. Adult Glioma Incidence and Survival by Race or Ethnicity in the United States From 2000 to 2014. JAMA Oncol 2018;4:1254-62.

9. Ochirjav E, Enkhbat B, Baldandorj T, et al. Reclassification of Mongolian Diffuse Gliomas According to the Revised 2016 World Health Organization Central 
Nervous System Tumor Classification. J Pathol Transl Med 2019;53:298-307.

10. Pekmezci M, Rice T, Molinaro AM, et al. Adult infiltrating gliomas with WHO 2016 integrated diagnosis: additional prognostic roles of ATRX and TERT. Acta Neuropathol 2017;133:1001-16.

11. Lu VM, O'Connor KP, Shah AH, et al. The prognostic significance of CDKN2A homozygous deletion in IDHmutant lower-grade glioma and glioblastoma: a systematic review of the contemporary literature. J Neurooncol 2020;148:221-9.

12. Ghasimi S, Wibom C, Dahlin AM, et al. Genetic risk variants in the CDKN2A/B, RTEL1 and EGFR genes are associated with somatic biomarkers in glioma. J Neurooncol 2016;127:483-92.

13. Anthony P, McArdle S, McHugh M. Tumor Treating Fields: Adjuvant Treatment for High-grade Gliomas. Semin Oncol Nurs 2018;34:454-64.

14. Hottinger AF, Pacheco P, Stupp R. Tumor treating fields: a novel treatment modality and its use in brain tumors. Neuro Oncol 2016;18:1338-49.

15. Stupp R, Taillibert S, Kanner AA, et al. Maintenance

Cite this article as: Lin Y, Chen B. Case report: tumortreating fields prolongs IDH-mutant anaplastic astrocytoma progression-free survival and pathological evolution to glioblastoma. Ann Transl Med 2021;9(24):1804. doi: 10.21037/ atm-21-4760
Therapy With Tumor-Treating Fields Plus Temozolomide vs Temozolomide Alone for Glioblastoma: A Randomized Clinical Trial. JAMA 2015;314:2535-43.

16. Shi W, Blumenthal DT, Oberheim Bush NA, et al. Global post-marketing safety surveillance of Tumor Treating Fields (TTFields) in patients with high-grade glioma in clinical practice. J Neurooncol 2020;148:489-500.

17. Onken J, Staub-Bartelt F, Vajkoczy P, et al. Acceptance and compliance of TTFields treatment among high grade glioma patients. J Neurooncol 2018;139:177-84.

18. Stupp R, Taillibert S, Kanner A, et al. Effect of TumorTreating Fields Plus Maintenance Temozolomide vs Maintenance Temozolomide Alone on Survival in Patients With Glioblastoma: A Randomized Clinical Trial. JAMA 2017;318:2306-16.

19. Kessler AF, Linsenmann T, Westermaier T, et al. Complete radiological response following subtotal resection in three glioblastoma patients under treatment with Tumor Treating Fields. Oncol Lett 2020;19:557-61.

(English Language Editor: J. Jones) 